\title{
Recurrence Quantification Analysis to Characterise the Heart Rate Variability Before the Onset of Ventricular Tachycardia
}

\author{
Niels Wessel ${ }^{1}$, Norbert Marwan ${ }^{1}$, Udo Meyerfeldt ${ }^{2}$, Alexander Schirdewan ${ }^{2}$, \\ Jürgen Kurths ${ }^{1}$ \\ ${ }^{1}$ University of Potsdam, Am Neuen Palais 10, PF 601553, \\ D-14415 Potsdam, Germany \\ ${ }^{2}$ Franz-Volhard-Hospital, Humboldt-University, Berlin, \\ Wiltbergstr. 50, D-13125 Berlin, Germany \\ \{meyerfeldt, schirdewan\} afvk-berlin.de
}

\begin{abstract}
Ventricular tachycardia or fibrillation (VT) as fatal cardiac arrhythmias are the main factors triggering sudden cardiac death. The objective of this recurrence quantification analysis approach is to find early signs of sustained VT in patients with an implanted cardioverter-defibrillator (ICD). These devices are able to safeguard patients by returning their hearts to a normal rhythm via strong defibrillatory shocks; additionally, they are able to store at least 1000 beat-to-beat intervals immediately before the onset of a lifethreatening arrhythmia. We study these 1000 beat-to-beat intervals of 63 chronic heart failure ICD patients before the onset of a life-threatening arrhythmia and at a control time, i.e. without VT event. We find that no linear parameter shows significant differences in heart rate variability between the VT and the control time series. However, the results of the recurrence quantification analysis are promising for this classification task.
\end{abstract}

\section{Introduction}

Implantable cardioverter defibrillators (ICD) are a safe and effective treatment for ventricular tachycardia or fibrillation (VT) [1-3]. These fatal cardiac arrhythmias are the main factors triggering sudden cardiac death. Therefore, an accurate identification of patients who are at high risk of sudden cardiac death is an important and challenging problem. Nowadays, third generation ICD offer not only important advances in arrhythmia treatment, but also permit the correct characterisation of the rhythm leading to intervention [4,5]. Additionally, they are able to store at least 1000 beat-to-beat intervals immediately before the onset of a life-threatening arrhythmia.

In this contribution we study the heart rate variability (HRV) of chronic heart failure ICD patients before the onset of a life-threatening arrhythmia and at a control time, i.e. without VT event. HRV parameters have been used to predict the mortality risk in patients with structural heart diseases [6,7]. Linear parameters only provide 
limited information about the underlying complex system, whereas nonlinear descriptions often suffer from the curse of dimensionality. This means that there are not enough points in the time series to reliably estimate these nonlinear measures. Therefore, we favour measures of complexity which are able to characterise quantitatively the dynamics even in rather short time series [8-10]. For ICD stored HRV data sets we found some evidence for predictability of VT in patients with a low number of ectopic beats [10]. In this investigation, we apply the concept of recurrence quantification analysis to find some precursors independently from the ectopy.

\section{Methods}

\subsection{Patients}

We studied the ICD stored 1000 beat-to-beat intervals before the onset of $131 \mathrm{VT}$ episodes and at 74 control intervals without VT in 63 ICD patients of the FranzVolhard-Hospital with severe congestive heart failure. No patient had received a class I or class III antiarrhythmic drug for $18 \pm 9$ months prior to the study. Time series including more than one episode nonsustained VT, episode of induced VT, or ventricular pacing are excluded from the analysis. To estimate the amount of ventricular premature beats we use an adaptive filtering algorithm for preprocessing, which tends to be superior to standard algorithms [11]. The beat-to-beat intervals of the VT at the end of the time series were removed from the tachograms so that we analysed only the dynamics occurring immediately prior to VT.

\subsection{Standard heart rate variability analysis}

To detect early signs of life-threatening VT, we applied a multiparametric analysis. Before starting the analysis, ventricular premature beats and artifacts usually should be removed from the time series to construct the so-called "normal-to-normal" beat time series (NN). We used the adaptive filtering algorithm [11] for preprocessing of the data. As a basically analysis a certain number of standard HRV parameters from time and frequency domain were calculated (e.g. $s d N N, p N N 50, L F / H F$ ) [12]. These standard parameters of HRV analysis are based on linear techniques. To classify dynamic changes in the time series, we present the following nonlinear concepts of recurrence quantification analysis outlined below.

\subsection{Recurrence quantification analysis}

Recurrence plots (RP) were firstly introduced to visualise time dependent behaviour of orbits in the phase space [13]. They represent the recurrence of the phase space trajectory to a state. The recurrence of states is a fundamental property of deterministic dynamical systems [14,15] - to quantify this effects Zbilut and Webber have introduced the recurrence quantification analysis (RQA) [16]. They define 
measures using recurrence point density, diagonal segments and paling in the recurrence plot, recurrence rate, determinism, averaged length of diagonal structures, entropy and trend. Two of these parameters turned out to be of interest in this paper: the determinism and entropy. Determinism is defined as the ratio of recurrence points forming diagonal structures to all recurrence points. The entropy denotes the Shannon entropy of the histogram of diagonal line segment lengths and reflects the complexity of the deterministic structure in the system. First promising applications of the RQA method to heart rate variability data are described in [17-19].

\subsection{The intermittency approach}

The described measures are using only diagonal structures in the RP. However, a RP contains more geometrical structures which may contain important information. Therefore, we introduce measures quantifying also horizontal and vertical structures, which give qualitative information about laminar behaviour and intermittency.

Analogous to the definition of averaged length of diagonal structures, we define the averaged length of vertical structures

$$
T T:=\sum_{v=v_{\min }}^{N} v \cdot P(v) / \sum_{v=v_{\min }}^{N} P(v)
$$

what we call trapping time TT. $P(v)$ is the probability distribution of vertical line of length $v$; the computation of $T T$ is realised for values of $v$ which exceed a minimal length $v_{\min }$. This was done to avoid the major influence of sojourn points, as described in [20]. TT emphasises parts of the RP with vertical lines represent laminar states in the system: A system consisting mainly of laminar (or trapped) states has a high TT, a system without laminar states has a very low $T T$.

Similar the definition of the determinism [16], we can compute the ratio of the recurrence points forming the vertical structures, the so called laminarity $L$ :

$$
L:=\sum_{v=v_{\min }}^{N} v \cdot P(v) / \sum_{v=1}^{N} v \cdot P(v)
$$

The laminarity decreases if the RP consists of more single recurrence points than vertical structures. Finally, the Shannon-entropy of the distribution of the vertical line lengths $P(v)$ is calculated which is a measure for the variability of the vertical structures. If there are vertical structures with varying lengths, the entropy will be high; a lot of vertical structures with the same (or similar) lengths will cause a small entropy. 


\section{Results}

\subsection{Standard heart rate variability}

We observed 131 VT episodes in 63 patients. Sixty-four episodes were not included because of atrial fibrillation, permanent pacing, incessant VT, incomplete storage of episodes, or storage artifacts. The remaining 67 VT episodes and 47 control series from 46 patients comprise the report. The mean VT cycle length was $310 \pm 53 \mathrm{~ms}$. Comparing the HRV parameters of all $67 \mathrm{VT}$ and 47 control time series, only the mean sinus rhythm cycle length showed differences, the time series leading to VT had a significantly shorter cycle length (meanNN $694.4 \pm 138.1 \mathrm{~ms}$ ), than the control time series (meanNN $760.8 \pm 140.2 \mathrm{~ms}$ ). The ectopy time, calculated as the sum of the coupling interval and the following pause of all premature beats, did not differ between the VT and control time series. The ectopy time of the VT group was $96.0 \pm 129.9 \mathrm{sec}$ according to $14 \%$ of all 1024 beat-to-beat intervals and $68.2 \pm 98.1 \mathrm{sec}$, according to $9 \%$ of the control time series ( $p>0.05$ ).

\subsection{Recurrence quantification analysis}

All parameters described in section 2.3. were calculated for the heart rate data before VT and at a control time. Embedding dimensions were chosen from 3 to 15 , the delay equal to 1 , radii from 5 to $50 \%$ for a relative scaling approach and radii ranging from 10 to $100 \mathrm{~ms}$ for absolute scaling. This investigation was a totally exploratory data analysis, we had to be aware of multiple testing problems in the statistical analysis. Nevertheless, we found some significant differences between both groups of time series (see table 1).

Table 1. Significant parameters in the RQA approach. The statistical analysis was based on the two-sided t-test as well the non-parametric Mann-Whitney-U-test.

\begin{tabular}{llll}
\hline Parameter & Embedding dimension & Radius & P \\
\hline Absolute scaling & & & \\
Entropy & 4 & 10 & $<0.05$ \\
Determinism & 12 & 20 & $<0.01$ \\
Determinism & 13 & 30 & $<0.05$ \\
Entropy & 14 & 40 & $<0.05$ \\
Determinism & 15 & 70 & $<0.05$ \\
\hline Relative scaling & & & \\
Determinism & 15 & 5 & $<0.01$ \\
Determinism & 15 & 10 & $<0.01$ \\
Determinism & $5 ; 6$ & 15 & $<0.05$ \\
Entropy & 10 & 20 & $<0.05$ \\
\hline
\end{tabular}

As one can see, significant results were obtained only for small radii and for high embedding dimensions. Figure 1 shows a typical example for the distribution of the 
RQA parameter. Significant differences were found because of zero recurrences in the control group 0. These results are in agreement with our findings in [10], intermittently decreased epochs of short HRV before VT lead to higher number of recurrences.

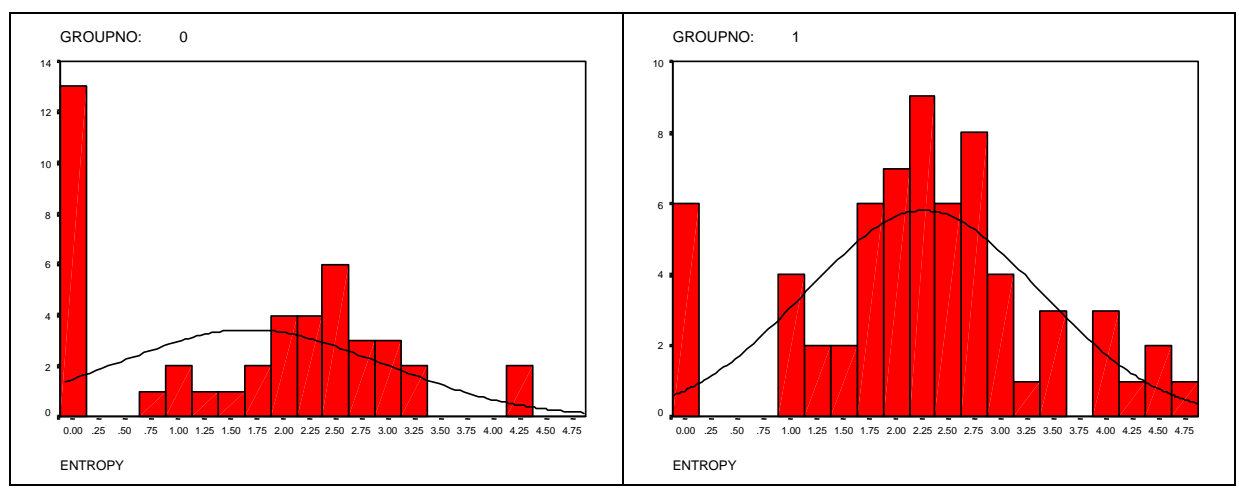

Fig. 1. The distributions of the entropy parameter for control group 0 as well as for VT group 1 calculated with an embedding dimension of 4 and a radius of 10 (absolute scaling).

Using the intermittency approach we could detect 8 VT time series out of all series which show extremely laminar behaviour. Non of the remaining data sets, neither control nor VT series, showed such short epochs with intermittently decreased HRV.

In a next step we tested the hypothesis if there is a correlation between the VT cycle length and some recurrence parameters before the onset of this VT. For small embedding dimensions and a fixed radius we found a increasing laminarity with increasing VT cycle length (see fig. 2). This means that serious VT with slow cycle length are characterised by a lower laminarity.

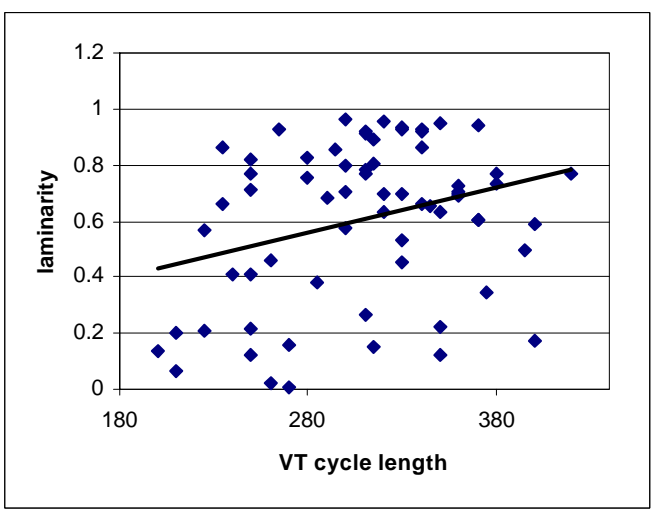

Fig. 2. The relation between the laminarity and the VT cycle length for an embedding dimension of 1 and a radius of 10 (absolute scaling). 


\section{Discussion}

We identified significant differences in the dynamic behaviour of beat-to-beat intervals between the VT and control time series by means of RQA parameters. They reflect increased short laminar phases with low variability in patients with congestive heart failure preceding the onset of VT.

Another important findings in this study were the significant $R R$ interval differences before the onset of slow and fast VT. The onset of slow VT was characterised by a significant increase in heart rate and an increase in laminarity. We assume that these differences illustrate a different role of autonomic regulation prior to the start of VT in both groups. Whereas slow VT began during sympathetic activation the fast arrhythmias were preceded by decreased heart rates and a low degree of laminarity.

Limitations of our study were the relatively small number of time series and the subsequently limited statistical analysis in terms of subdivisions concerning age, sex, and heart disease. Thus, these results must be validated with a larger data base. Our data offer the possibility of developing automatic ICD algorithms based on nonlinear dynamic HRV parameters.

\section{Acknowledgements}

We want to thank Alessandro Giuliani and Joseph Zbilut for helpful discussions.

\section{References}

1. Mirowski, M., Reid, P.R., Mower, M.M., Watkins, L., Gott, V.L., Schauble, J.F., Langer, A., Heilman, M.S., Kolenik, S.A., Fischell, R.E., Weisfeldt, M.L.: Termination of malignant ventricular tachyarrhythmias with an implanted automatic defibrillator in human beings. N Engl J Med 303 (1980) 322-324

2. Grimm, W., Flores, B.T., Marchlinski, F.E.: Shock occurence and survival in 241 patients with implantable cardioverter-defibrillator therapy. Circulation 87 (1993) 18880-18888

3. The antiarrhythmic versus implantable defibrillators (AVID) investigators: A comparison of antiarrhythmic drug therapy with implantable defibrillators in patients resuscitated from near fatal ventricular arrhythmias. N Engl J Med 337 (1997) 1576-1583

4. Hook, B.G., Callans, D.J., Kleiman, R.B., Flores, B.T., Marchlinski, F.E.: Implantable cardioverter-defibrillator therapy in the absence of significant symptoms. Circulation 87 (1993) 1897-1906

5. Gronefeld, G.C., Mauss, O., Li, Y.G., Klingenheben, T., Hohnloser, S.H.: Association between atrial fibrillation and appropriate implantable cardioverter defibrillator therapy: results from a prospective study. J Cardiovasc Electrophysiol 11 (2000) 1208-1214

6. Kleiger, R.E., Miller, J.P., Bigger, J.T., Moss, A.: Decreased heart rate variability and its association with increased mortality after acute myocardial infarction. Am J Cardiol 59 (1987) 256-262 
7. Tsuji, H., Larson, M.G., Venditti, F.J. Jr, Manders, E.S., Evans, J.C., Feldman, C.L., Levy, D.: Impact of reduced heart rate variability on risk for cardiac events. The Framingham Heart Study. Circulation 94 (1996) 2850-2855

8. Kurths, J., Voss, A., Witt, A., Saparin, P., Kleiner, H.J., Wessel, N.: Quantitative analysis of heart rate variability. Chaos 5 (1995) 88-94

9. Voss, A., Kurths, J., Kleiner, H.J., Witt, A., Wessel, N., Saparin, P., Osterziel, K.J., Schurath, R., Dietz, R.: The application of methods of non-linear dynamics for the improved and predictive recognition of patients threatened by sudden cardiac death. Cardiovasc Res 31 (1996) 419-433

10. Wessel, N., Ziehmann, Ch., Kurths, J., Meyerfeldt, U., Schirdewan, A., Voss, A.: Shortterm Forecasting of Life-threatening Cardiac Arrhythmias based on Symbolic Dynamics and Finite-Time Growth Rates. Phys Rev E 61 (2000) 733-739

11. Wessel, N., Voss, A., Malberg, H., Ziehmann, Ch., Voss, H.U., Schirdewan, A., Meyerfeldt, U., Kurths, J.: Nonlinear analysis of complex phenomena in cardiological data. Herzschr Elektrophys 11 (2000) 159-173

12. Heart rate variability: standards of measurement, physiological interpretation and clinical use. Task Force of the European Society of Cardiology and the North American Society of Pacing and Electrophysiology. Circulation 93 (1996) 1043-1065

13. Eckmann, J.-P., Kamphorst, S.O., Ruelle, D.: Recurrence plots of dynamical systems. Europhys Lett 4 (1987) 973-977

14. Argyris, J.H., Faust, G., Haase, M.: An exploration of Chaos. North Holland, Amsterdam, 1994

15. Ott, E.: Chaos in dynamical systems. University Press, Cambridge, 1993

16. Zbilut, J.P., Webber Jr., C.L.: Embeddings and delays as derived from quantification of recurrence plots. Phys. Lett. A 171 (1992) 199-203

17. Giuliani, A., Piccirillo, G., Marigliano, V., Colosimo, A.: A nonlinear explanation of aginginduced changes in heartbeat dynamics. Am J Physiol 275 (1998) H1455-H1461

18. Gonzalez, J. J., Cordero, J. J., Fe ria, M., Pereda, E.: Detection and sources of nonlinearity in the variability of cardiac R-R intervals and blood pressure in rats. Am J Physiol Heart Circ Physiol 279 (2000) H3040-H3046

19. Censi, F., Barbaro, V., Bartolini, P., Calcagnini, G., Michelucci, G. F., Cerutti, S.: Recurrent patterns of atrial depolarization during atrial fibrillation assessed by recurrence plot quantification. in: Annals of Biomedical Engineering 28 (2000), 61-70

20. Gao, J., Cai, H.: On the structures and quantification of recurrence plots. Physics Letters A 270 (2000), $75-87$ 